\title{
P WAVE DISPERSION AND CARDIAC TROPONIN I CONCENTRATION IN CANINE VISCERAL LEISHMANIASIS*
}

\author{
Deniz NAKİPOĞLU ${ }^{1}$, Kerem URAL \\ ${ }^{1}$ Adnan Menderes University, Faculty of Veterinary, Department of Internal Medicine, Isikli, 09016, \\ Aydin, TURKEY.
}

Geliş Tarihi: 20.04.2017 Kabul Tarihi: 20.06.2017

Makale Kodu:306728

Bu makaleye atıfta bulunmak için/To cite this article:

Nakipoğlu D, Ural K. P Wave Dispersion and Cardiac Troponin I Concentration In Canine Visceral Leishmaniasis. MAE Vet Fak Derg. 2018;3(1):1-16.

\begin{abstract}
In the present study, the aim was to clinically evaluate the probable cardiac damage in dogs with CVL according to its stage via measuring atrial conduction time by use of $\mathrm{Pd}$ determined within computerized electrocardiography and cardiac troponin I (CTnI) level.

A total of 18 diseased dogs, of both sexes and various ages, referred with one or more of the clinical findings such as hypertrichosis, periocular alopecia, weight loss, onychogryphosis, skin lesions (severe scaling, exfoliative dermatitis compatible with alopecia) and/or anorexia, lymphadenopathy, hepatosplenomegaly were involved. In the healthy control group for comparison and in dogs with CVL to determine presence, nature and level of cardiac damage, the evaluations were performed with computerized 12 -lead ECG device $[(1 \mathrm{mV} / \mathrm{cm}$ amplitude in resting and $50 \mathrm{~mm} / \mathrm{sec}$ ) (Pd measurement)]. Serum CTnI concentrations were measured by using species specific commercial test kit.

High levels of cTnI concentration were detected in 10 of 18 dogs infected CVL with all polysymptomatic dogs. In all cases of the control group, cTnI levels were in the reference range $[<0.03 \mathrm{ng} / \mathrm{dL}]$. Even the comparison of each groups, no statistically significance $(\mathrm{p}>0.05)$ was found between CVL positive and control dogs. Mean \pm standard deviation of Pd values were 22.76 $\pm 3.12,22.03 \pm 0.80,22.73 \pm 0.80$ and $25.67 \pm 1.41$ in the control group, asymptomatic group, oligosymptomatic group, and polysymptomatic group, respectively. In comparison between groups, polysymptomatic group was significantly different than control $(\mathrm{p}=0.026)$, asymptomatic $(\mathrm{p}=0.012)$ and oligosymptomatic $(\mathrm{p}=0.027)$ groups.

Although a statistically significant difference was not found between CVL positive and control dogs in the present study, it was suggested that the individual increase may be associated with myocarditis due to disease. Besides, it may be claimed that the mean Pd values determined in especially polysymptomatic dogs was higher compared to the control group, whereas this may be accepted in the reference ranges based on mean Pd values reported in healthy dogs. However considering infected dogs population as 6 in each group, it may be safely claimed that further investigations regarding greater number of cases the may be warranted.
\end{abstract}

Keywords: Canine Visceral Leishmaniasis, Cardiac Troponin I, Dog, P Wave Dispersion, ECG

* The study was summarized from the Master of Science Thesis of Nakipoğlu D. Graduated from Adnan Menderes University The Graduate School Of Health Sciences Veterinary Master Of Science Program Thesis, Aydın, 2016 with project number:VTF-14036 financially supported by BAP unit Adnan Menderes University

İletişim / Correspondence

Adnan Menderes University, Faculty of Veterinary, Department of Internal Medicine, Isikli, 09016, Aydın, TURKEY.

$8+9$

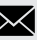

uralkerem@gmail.com

ORCIDDeniz NAKIPOĞLU:0000-0001-9699-1504

Kerem URAL:0000-0003-1867-7143 


\section{CANINE VISCERAL LEISHMANIASIS'TE P DALGA DISPERSIYONU VE KARDIYAK TROPONIN I KONSANTRASYONU}

\section{ÖZ}

$\mathrm{Bu}$ çalışmada, CVL'li köpeklerde evrelerine göre muhtemel kalp hasarının bilgisayarlı elektrokardiyografi ile atriyal iletim süresinin ölçülmesi yoluyla Pd'nin kullanımı ve kardiyak troponin I (CTnI) seviyelerinin ölçülmesiyle, klinik olarak değerlendirilmesi amaçlanmıştır.

Hipertrikozis, perioküler alopesi, kilo kaybı, onikogripozis, lenfadenopati, hepatosplenomegali ve deri lezyonları (şiddetli kabuklanma, alopesi ile uyumlu eksfoliatif dermatitis) ve/veya anoreksiya gibi bir veya daha fazla klinik bulgunun bulunduğu her iki cinsiyetten ve farklı yaşlardan toplam 18 hasta köpek çalışma kapsamına alındı. Kardiyak hasarın varlığı, niteliği ve seviyesini belirlemek için CVL'li köpeklerde, karşılaştırma için sağlıklı kontrol grubunda ve, bilgisayarlı 12 kanallı EKG cihazı [dinlenme ve $50 \mathrm{~mm} / \mathrm{sn}$ 'de $1 \mathrm{mV} / \mathrm{cm}$ amplitüd] ( Pd ölçümü)] kullanıldı. Serum CTnI konsantrasyonları, türe özgü ticari test kiti kullanılarak ölçüldü.

CVL ile polisemptomatik olarak enfekte 18 köpekten 10'unda yüksek düzeyde cTnI konsantrasyonu tespit edildi. Kontrol grubunun tüm olgularında cTnI seviyeleri referans aralıkta $[<0.03 \mathrm{ng} / \mathrm{dL}]$ idi. Her bir grubun karşılaştırılmasında, CVL pozitif ve kontrol köpekleri arasında istatistiksel olarak anlamlı bir fark bulunamadı ( $>$ > 0.05). Pd değerlerinin ortalama \pm standart sapması sirasıyla kontrol grubu, asemptomatik grup, oligosemptomatik grup ve polisemptomatik grupta $22.76 \pm 3.12,22.03 \pm 0.80,22.73 \pm 0.80$ ve $25.67 \pm 1.41$ idi. Gruplar arasında karşılaştırıldığında, polisemptomatik grup kontrol grubundan $(\mathrm{p}=0.026)$, asemptomatik $(\mathrm{p}=0.012)$ ve oligosemptomatik $(\mathrm{p}=0.027)$ gruptan anlamlı farkl1lık gösterdi.

$\mathrm{Bu}$ çalışmada CVL pozitif ve kontrol olgular arasında cTnI seviyeleri açısından istatistiksel olarak anlam11 fark bulunmasa da, bireysel artışlar hastalığa bağlı olarak miyokardit ile ilişkili olabileceğini göstermektedir. Ayrıca, özellikle polisemptomatik köpeklerde saptanan ortalama Pd değerlerinin kontrol grubuna göre daha yüksek olduğu ortaya konulsa da bu durum sağl1klı köpeklerde bildirilen ortalama Pd değerlerine dayalı referans aralıklarında kabul edilebilmektedir. Buna karşın, enfekte köpeklere ait her bir grupta 6 olgu olduğu düşünülürse, daha fazla sayıdaki olgunun araştırılmasının faydalı olabileceği öne sürülebilir.

Anahtar Kelimeler: Canine Visceral Leishmaniasis, Kardiyak Troponin I, Köpek, P dalga dispersiyonu, EKG 


\section{INTRODUCTION}

Canine Visceral Leishmaniasis (CVL), as a well recognized parasitic and zoonotic disease, is caused by protozoan of the genus Leishmania. The etiological agent may be transmitted to the dog via Phlebotomus species bite. Among Mediterranean location, L. infantum is the species usually implicated in CVL, a systemic form of the disease presenting involvement of multiple organs such as kidneys, liver, lymph nodes, bone marrow, spleen and skin $(1,2)$. Although clinical signs related to cardiac disease were not frequently reported $(3,4)$, myocaridal lesion might exist (5-7). Besides Leishmania parasite may be detected in cardiac tissue (3$6,8)$.

Given canine myocarditis in relation with L. infantum infection has already been determined $(6,9)$, with pathological changes composed of degeneration and necrosis of cardiomyocytes and interstitial infiltration $(5,8)$, it should not be unwise to draw conclusion that cardiac involvement in dogs with CVL of different stages should be investigated with electrocaridographic and cardiac troponin I investigations, which was the puposes of this study.

\section{MATERIALS AND METHODS}

\section{Animal material}

A total of 24 dogs, from both sexes and different ages with presenting none, one or more of the complaints of hypotrichosis, periocular alopecia, weight loss, onichogrypphosis, skin lesions (scalling, alopecia in association with exfoliative dermatitis) and/ oranorexia, lymphadenopathy, hepatosple- nomegalia. The study protocol was approved by the institutional laboratory animals ethics committee of Adnan Menderes University, Local Ethics Committee HADYEK (with no: 64583101/2013/060). Informed written consent was obtained from all of the owners for participating of their animals in this study.

\section{Indirect Floresan Antibody Test (IFAT)}

This test was applied at Adnan Menderes University, Faculty of Medicine, Parasitology Department as previously described (10). Bright yellow green fluorescence were deemed positive, pale/none yellow-green flourescence were denoted as negative. Highest serum dilution fluoresced were deemed antibody titer regarding related sample. Immuno fluorescent antibody titer 1/64 and above for samples were denoted as positive against CVL (10).

\section{Classification to the groups}

Following IFAT analysis allowing us to diagnose precise diagnosis of CVL, dogs were further enrolled into 4 different groups ( $n=6$ in each). The dogs were denoted oligosymptomatic [in group I, solely one or two clinical signs (12-14) present] (Figure 1), polisymptomatic (in group II, even if presented three or more of the clinical signs in association with CVL) (Figure 2), and asymptomatic (in group III, even if free from clinical symptoms) (Figure 3), as was also described (14-16). Group IV were composed of IFAT negative, non CVL, healthy cases. 


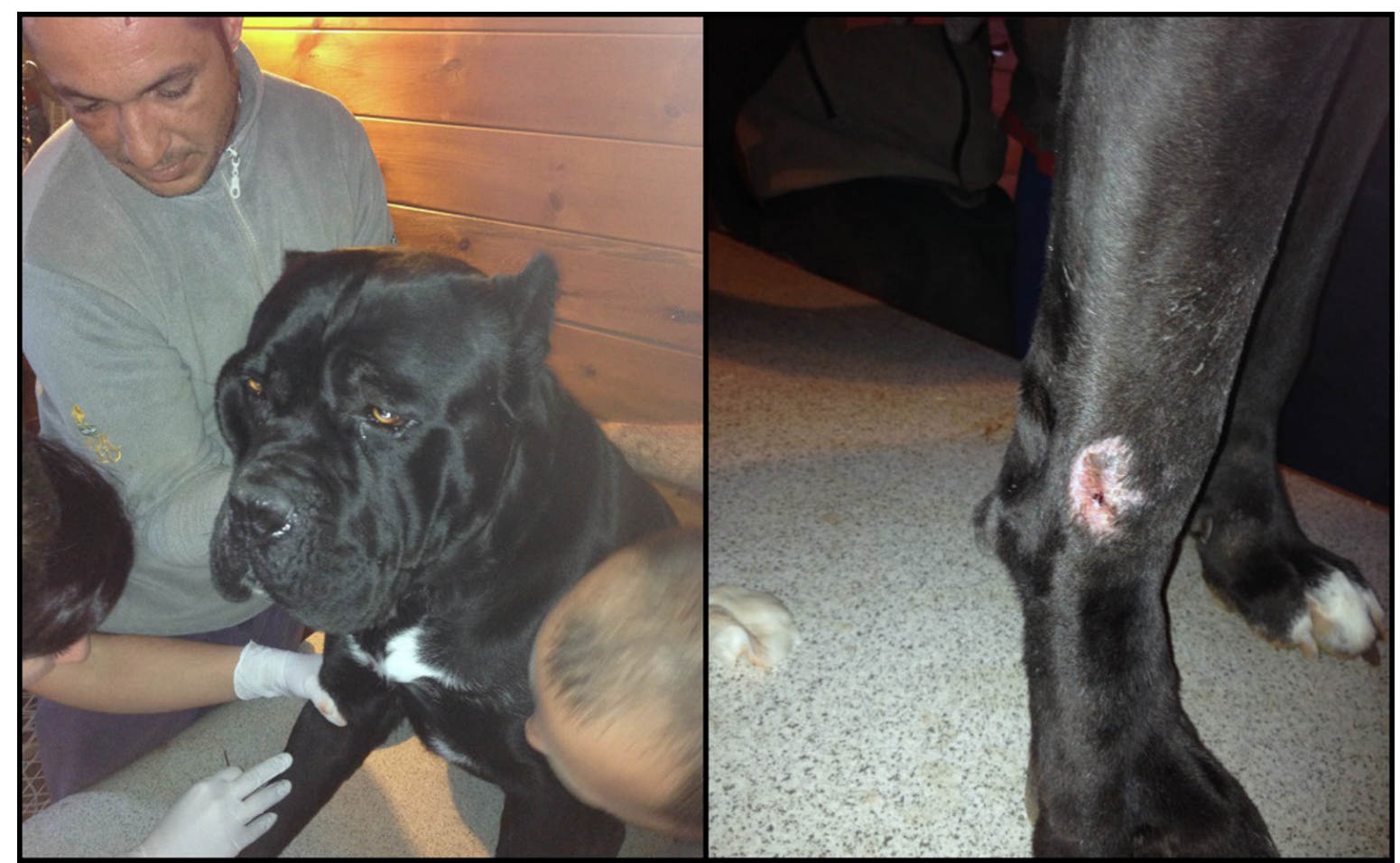

Figure 1. Oligosymptomatic CVL infected Presa Canario dog presenting ertyhematosus based demarcative and ulcerated lesion.

ECG examination with special reference stored as direct electronic signals, every half to $\mathbf{P d}$ minute by use of computer software BTL. All dogs were subjected to ECG in standing Besides computerized system within the

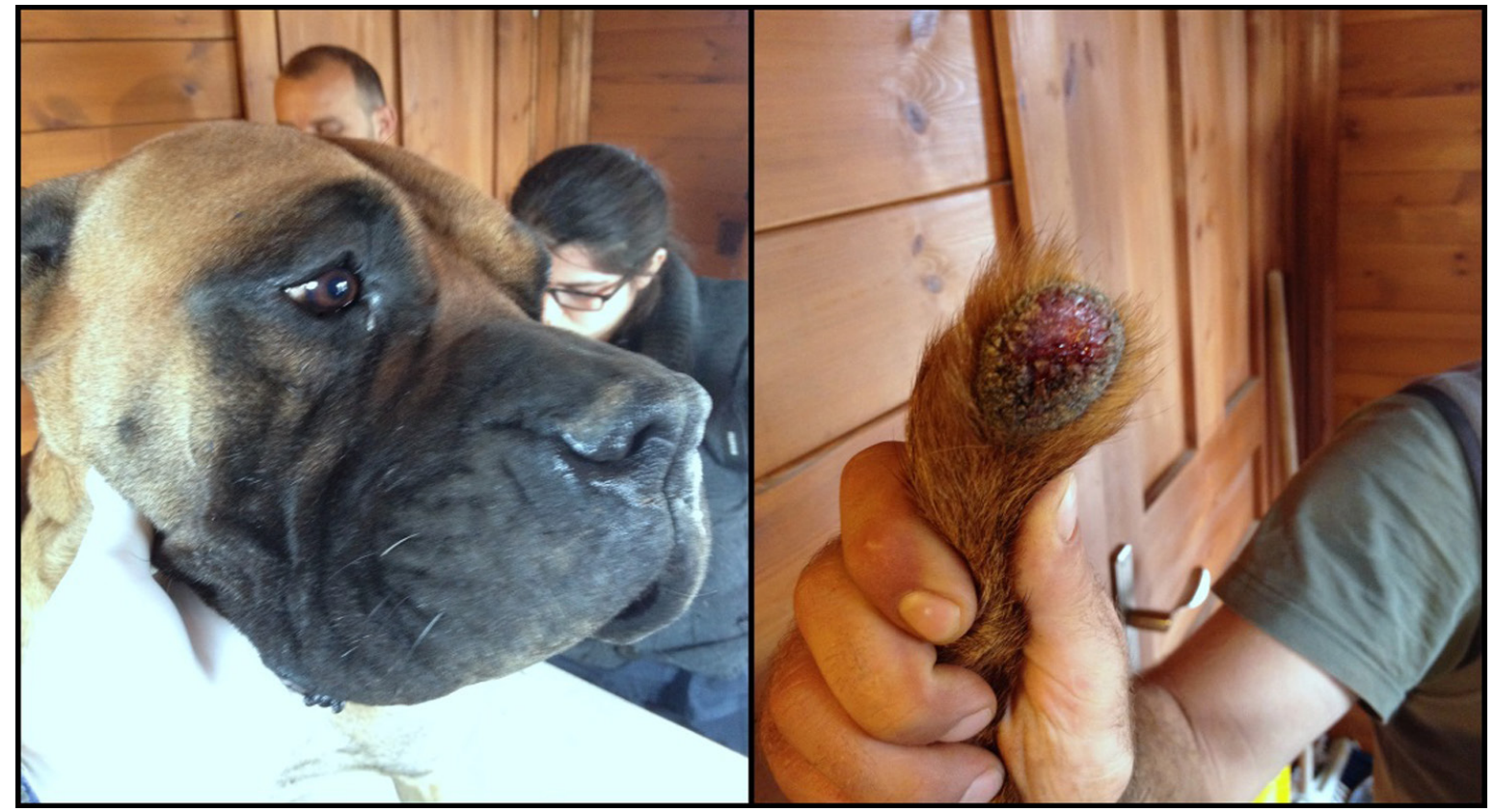

Figure 2. Polisymptomatic CVL infected Presa Canario dog. There was no traumatic history, with an ulcerated, haemorrhagic, crusted and alopecic lesion on tail.

position on BTL MT08 equipped with ECG record, permitted us to prevent the computerized 12 lead. The ECG signals were interference of muscles on the records and 
besides eliminate probable artefacts, as was also described previously (17). The BTL

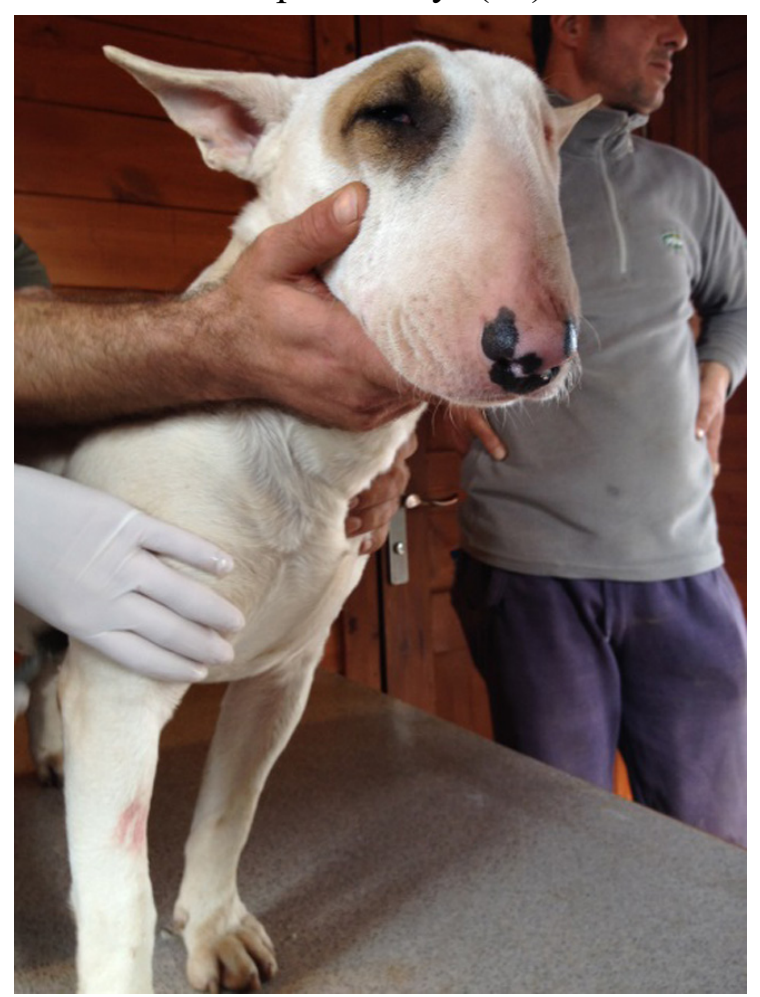

Figure 3. Asymptomatic Bullterrier dog with CVL. space), V2 - beneath the left of the sternum, V4 -on to the left at the costochondrial junction (at the 6th intercostal space) (17, 18). The record was analyzed with at most care in an attempt to analyzed on $9 \mathrm{ECG}$ leads (I, II, III, IV, aVR, aVL, aVF, V1, V2, V4) at five cardiac cycles (17) (Figure 4). Each established lead presented the duration of P-wave, calculated as the distance among the onset (positive/negative deflection from the isoelectric line) and the offset (return to the isoelectric line) with precision to $1 \mathrm{~ms}$. This was followed bycalculation of Pmin (minimum) and Pmax(maximum)values of $\mathrm{P}$-wave. The Pd was measured within the difference between Pmax and Pmin and to those of average from 5 measurements have been used (figure 5) (17-20).

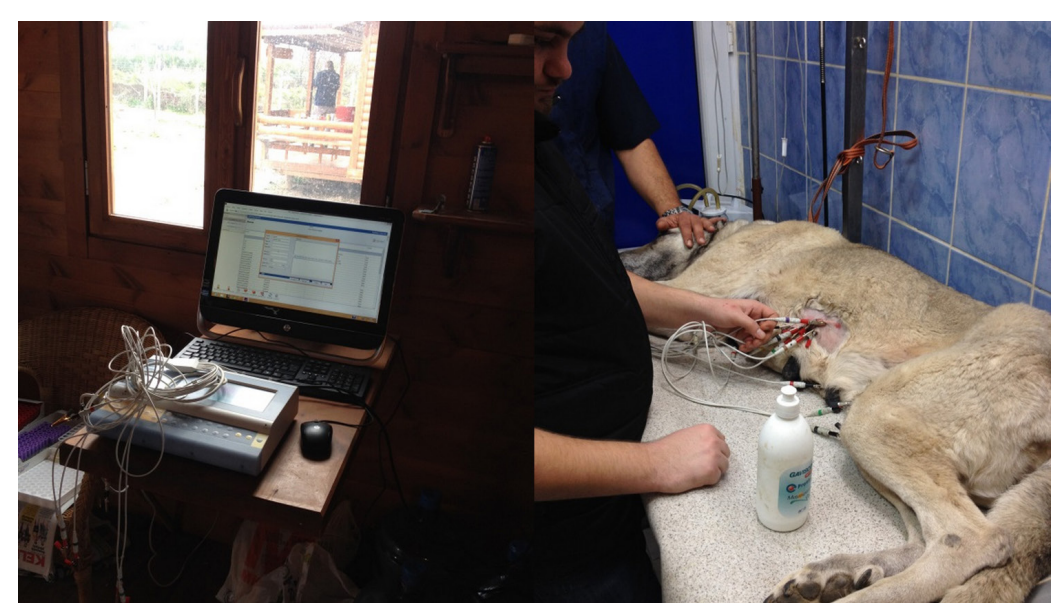

Figure 4. Preparation for the ECG application under field conditions

system is capable of enlarging the record 200 times during usage of the computer display. Necessary electrodes such as red (right arm); yellow (left arm); black (right leg); and green (left leg) were introduced respectively. The precordial leads were subjected as: V1 on right of sternum (at the 5th intercostal

\section{Laboratory applications}

\section{Physical and hematologic examinations}

In animals infected with CVL, history, age, gender, physical examination and laboratory findings were recorded (Table 1-5). In all cases, immediately prior to any application, in an attempt to perform IFAT and serum 
biochemical analysis, blood samples were red at $80^{\circ} \mathrm{C}$ till analysis.

withdrawnfrom vena cephalica antebrachii into anticoagulant tubes $(5 \mathrm{ml})$ for a single application and were evaluated.

\begin{tabular}{|c|c|c|c|c|c|c|c|c|c|c|}
\hline$\|$ & $\|$ & ovR & an & aVF & C1 & $\mathrm{C}_{2}$ & $c_{4}$ & max & $P_{\text {max }} \cdot P_{\min }$ & \\
\hline & & & 104 & & 94 & & & 122 & 28 & 1 complex \\
\hline & & & & & 118 & 124 & 122 & 124 & 6 & 2 complex \\
\hline & & & & & 88 & 104 & 102 & 104 & 38 & 3 cannlex \\
\hline & & & 108 & 102 & 100 & 96 & 84 & 108 & 24 & 4 complex \\
\hline & & & & & 102 & 90 & 88 & 102 & 38 & 5 camplex \\
\hline
\end{tabular}

Pd 27

Figure 5. $\mathrm{P}$ wave dispersion $(\mathrm{Pd})$ measurement. University of Adnan Menderes, Faculty of Veterinary Medicine Archive.

\section{Serum cardiac troponin I concentrations}

Serum cTnI concentrations were evalauted comperatively (in healthy group) and for de-

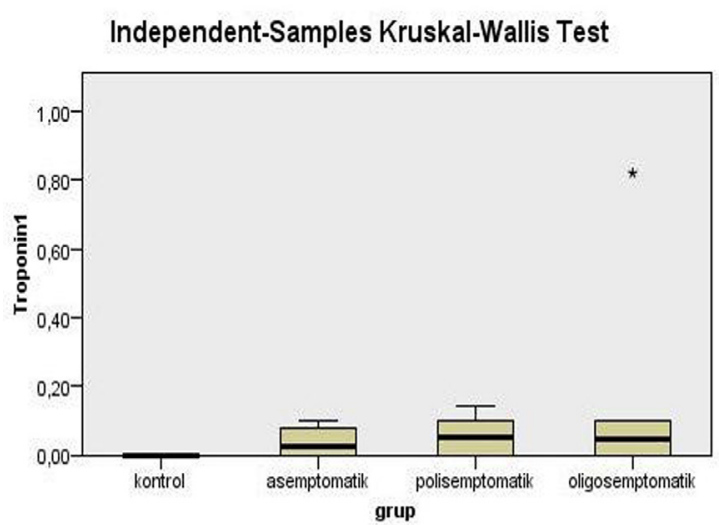

Figure 6. Intergroup distribution box plot graph of cardiac troponin I concentration (Kruskal-Wallis test $\mathrm{p}=0.145$ )

tecting severity, presence and concentrations for probable mycoardial injury (in CVL infected dogs). Dog specific commercially available ELISA test kits (Kamiya Biomedical Company - Dog Cardiac Troponin-I, High Sensitive ELISA Cat. No: KT-640) were used in a private laboratory (Bilim Laboratory, İstanbul, TURKEY). Samples were sto-

\section{Statistical analysis}

Statistical analysis were performed by use of SPSS statistical software package (version 22; SPSS Inc. Chicago, IL). Both control and infected groups data were not normally distributed. Following logaritmic transformation, as data were abnormally distributed, non-parametric Kruskal-Wallis test andpaired way post-hoc were used for comparison.

\section{RESULTS}

\section{cTnI analysis results}

cTnI analysis were presented on tables 1-5. Among CVL infected dogs, 10/18 presented elevated levels. Interestingly $3 / 6$ of asymptomatic and oligosymptomatic dogs, and 4 out of 6 polisymptomatic dogs presented higher cTnI concentrations. Among control groups 6/6 had cTnI concentrations in reference ranges $[<0,03$ ng/dL]. Min-max along with mean \pm st. dev. (ng/dL) values were shown in table 5. As was also shown in table 5, there was no statistical

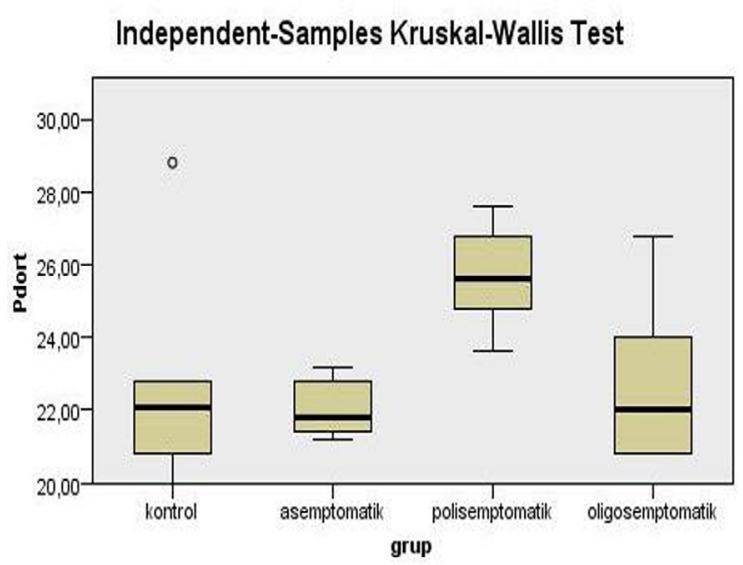

Figure 7. Pd result of intergroup distribution box on the chart (boxplot) (Kruskal-Wallis test $p=0.042$ )

significance $(p>0,05)$ between control and infected groups (Figure 6). 
Table 1. Demographic data regarding control group; breed, gender (sex),age distribution, IFAT titers, cTnI concentration and Pd analysis.

\begin{tabular}{|c|c|c|c|c|c|c|c|c|c|c|c|c|c|c|c|}
\hline \multicolumn{16}{|c|}{ CONTROL GROUP } \\
\hline \multirow[b]{2}{*}{$\dot{z}$} & \multirow[b]{2}{*}{ 苞 } & \multirow[b]{2}{*}{ 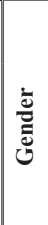 } & \multirow{2}{*}{ 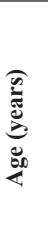 } & \multirow[b]{2}{*}{ 氞 } & \multirow{2}{*}{ 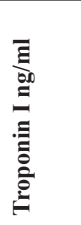 } & \multicolumn{10}{|c|}{ P- wave dispersion } \\
\hline & & & & & & I & II & III & aVR & aVL & aVF & $P$ max & $P \min$ & Pd & mean Pd \\
\hline \multirow{5}{*}{1} & \multirow{5}{*}{ 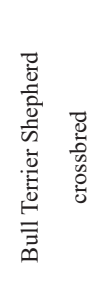 } & & & \multirow{5}{*}{ 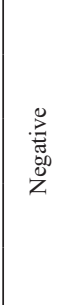 } & \multirow{5}{*}{ 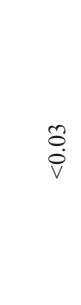 } & 58 & 50 & 46 & 56 & 52 & 74 & 74 & 46 & 28 & \multirow{5}{*}{22} \\
\hline & & & & & & 60 & 70 & 50 & 58 & 68 & 50 & 70 & 50 & 20 & \\
\hline & & & & & & 50 & 38 & 48 & 40 & 62 & 58 & 62 & 38 & 24 & \\
\hline & & & & & & 48 & 54 & 56 & 56 & 58 & 66 & 66 & 48 & 18 & \\
\hline & & & & & & 66 & 54 & 50 & 70 & 62 & 56 & 70 & 50 & 20 & \\
\hline \multirow{5}{*}{2} & \multirow{5}{*}{ 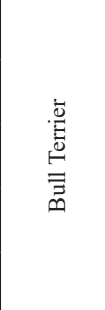 } & \multirow{5}{*}{ 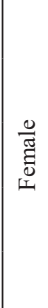 } & \multirow{5}{*}{$m$} & \multirow{5}{*}{ 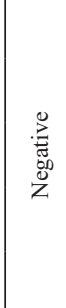 } & \multirow{5}{*}{$\stackrel{\tilde{\theta}}{\dot{v}}$} & 53 & 58 & 64 & 50 & 69 & 50 & 69 & 50 & 19 & \multirow{5}{*}{22,8} \\
\hline & & & & & & 37 & 48 & 74 & 42 & 58 & 53 & 74 & 37 & 37 & \\
\hline & & & & & & 32 & 53 & 42 & 40 & 63 & 42 & 63 & 32 & 31 & \\
\hline & & & & & & 48 & 53 & 64 & 48 & 48 & 48 & 64 & 48 & 16 & \\
\hline & & & & & & 53 & 42 & 53 & 53 & 53 & 53 & 53 & 42 & 11 & \\
\hline \multirow{5}{*}{3} & \multirow{5}{*}{ 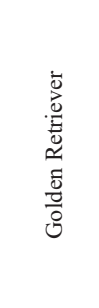 } & \multirow{5}{*}{$\frac{0}{\frac{\pi}{\pi}}$} & & & & 74 & 64 & 86 & 74 & 68 & 78 & 86 & 64 & 22 & \\
\hline & & & & & & 68 & 74 & 66 & 68 & 78 & 58 & 78 & 58 & 20 & \\
\hline & & & $\stackrel{n}{\sim}$ & 焉 & $\begin{array}{l}\tilde{o} \\
\hat{\theta}\end{array}$ & 48 & 64 & 64 & 64 & 68 & 66 & 68 & 48 & 20 & 20 \\
\hline & & & & & & 64 & 76 & 64 & 62 & 80 & 64 & 80 & 62 & 18 & \\
\hline & & & & & & 56 & 68 & 70 & 66 & 76 & 72 & 76 & 56 & 20 & \\
\hline & & & & & & 74 & 54 & 48 & 56 & 68 & 60 & 74 & 48 & 26 & \\
\hline & $\stackrel{\circ}{\square}$ & & & & & 58 & 52 & 64 & 62 & 82 & 58 & 82 & 52 & 30 & \\
\hline 4 & $\underset{\widetilde{J}}{\widetilde{\Xi}}$ & 吕 & $\stackrel{n}{m}$ & 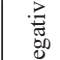 & $\stackrel{m}{\dot{\theta}}$ & 68 & 56 & 66 & 58 & 78 & 66 & 78 & 56 & 22 & 20,8 \\
\hline & $\equiv$ & & & & & 64 & 54 & 62 & 66 & 56 & 66 & 66 & 54 & 12 & \\
\hline & & & & & & 62 & 56 & 58 & 68 & 70 & 62 & 70 & 56 & 14 & \\
\hline & & & & & & 66 & 58 & 54 & 72 & 68 & 74 & 74 & 54 & 20 & \\
\hline & $\cong$ & & & & & 68 & 60 & 58 & 80 & 58 & 58 & 80 & 58 & 22 & \\
\hline 5 & $\underbrace{\mathbb{E}}_{\widetilde{\Xi}}$ & 岢 & 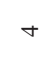 & 鴶 & $\hat{\tilde{\varphi}}$ & 58 & 72 & 50 & 64 & 56 & 66 & 72 & 50 & 22 & 22,8 \\
\hline & $\stackrel{2}{2}$ & & & & & 64 & 60 & 58 & 78 & 64 & 48 & 78 & 48 & 30 & \\
\hline & & & & & & 68 & 56 & 48 & 64 & 62 & 68 & 68 & 48 & 20 & \\
\hline & & & & & & 48 & 53 & 53 & 48 & 58 & 53 & 58 & 48 & 10 & \\
\hline & & & & & & 64 & 85 & 48 & 53 & 64 & 53 & 85 & 48 & 37 & \\
\hline 6 & త్ & $\frac{0}{\pi}$ & $m$ & 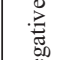 & $\stackrel{\text { ô }}{\circ}$ & 48 & 48 & 48 & 58 & 69 & 58 & 69 & 48 & 21 & 22,2 \\
\hline & 离 & & & & & 42 & 53 & 64 & 69 & 58 & 48 & 69 & 42 & 27 & \\
\hline & & & & & & 53 & 64 & 48 & 64 & 64 & 53 & 64 & 48 & 16 & \\
\hline
\end{tabular}


Table 2. Demographic data regarding asymptomatic control group; breed, gender (sex), age distribution, IFAT titers, cTnI concentration and Pd analysis.

\begin{tabular}{|c|c|c|c|c|c|c|c|c|c|c|c|c|c|c|c|}
\hline \multicolumn{16}{|c|}{ ASYMPTOMATIC GROUP } \\
\hline \multirow[b]{2}{*}{$\dot{z}$} & \multirow[b]{2}{*}{ 苞 } & \multirow[b]{2}{*}{ Ũ } & \multirow[b]{2}{*}{ 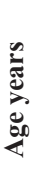 } & \multirow[b]{2}{*}{ 氞 } & \multirow{2}{*}{ 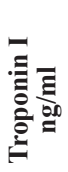 } & \multicolumn{10}{|c|}{ P- wave Dispersion } \\
\hline & & & & & & I & II & III & aVR & aVL & aVF & $P$ max & $P \min$ & P dispersion & $\begin{array}{c}\text { mean } \\
\text { Pd }\end{array}$ \\
\hline \multirow{5}{*}{1} & \multirow{5}{*}{ 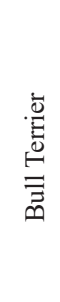 } & \multirow{5}{*}{ 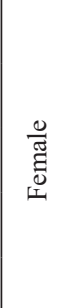 } & \multirow{5}{*}{$\stackrel{n}{n}$} & \multirow{5}{*}{$\stackrel{\mathscr{\swarrow}}{\stackrel{n}{S}}$} & \multirow{5}{*}{$\begin{array}{l}\infty \\
\stackrel{0}{0}\end{array}$} & 53 & 48 & 58 & 64 & 58 & 64 & 64 & 48 & 16 & \multirow{5}{*}{21,4} \\
\hline & & & & & & 58 & 64 & 42 & 58 & 58 & 58 & 64 & 42 & 22 & \\
\hline & & & & & & 53 & 58 & 58 & 64 & 53 & 69 & 69 & 53 & 16 & \\
\hline & & & & & & 64 & 58 & 58 & 53 & 48 & 69 & 69 & 48 & 21 & \\
\hline & & & & & & 42 & 69 & 58 & 53 & 53 & 74 & 74 & 42 & 32 & \\
\hline \multirow{5}{*}{2} & \multirow{5}{*}{ 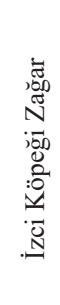 } & \multirow{5}{*}{ 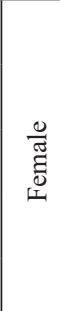 } & \multirow{5}{*}{$\begin{array}{l}n \\
\tilde{r}^{\prime}\end{array}$} & \multirow{5}{*}{ 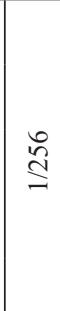 } & \multirow{5}{*}{$\overrightarrow{0}$} & 98 & 102 & 80 & 96 & 92 & 74 & 102 & 74 & 28 & \multirow{5}{*}{22} \\
\hline & & & & & & 48 & 66 & 72 & 58 & 68 & 48 & 72 & 48 & 24 & \\
\hline & & & & & & 62 & 66 & 48 & 50 & 68 & 46 & 68 & 46 & 22 & \\
\hline & & & & & & 64 & 50 & 66 & 56 & 64 & 70 & 70 & 50 & 20 & \\
\hline & & & & & & 64 & 56 & 70 & 54 & 68 & 64 & 70 & 54 & 16 & \\
\hline \multirow{5}{*}{3} & \multirow{5}{*}{ 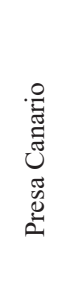 } & \multirow{5}{*}{ 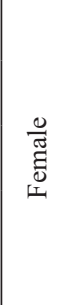 } & & & & 48 & 48 & 53 & 42 & 58 & 48 & 58 & 42 & 16 & \\
\hline & & & & & & 58 & 48 & 53 & 42 & 48 & 42 & 58 & 42 & 16 & \\
\hline & & & $\stackrel{n}{*}$ & $\stackrel{t}{=}$ & $\begin{array}{l}\hat{\theta} \\
\hat{\theta}\end{array}$ & 42 & 53 & 48 & 48 & 48 & 64 & 64 & 42 & 22 & 21,6 \\
\hline & & & & & & 48 & 42 & 69 & 53 & 53 & 53 & 69 & 42 & 27 & \\
\hline & & & & & & 42 & 42 & 53 & 58 & 53 & 69 & 69 & 42 & 27 & \\
\hline & & & & & & 76 & 74 & 74 & 74 & 68 & 80 & 80 & 68 & 12 & \\
\hline & Еี & & & & & 58 & 76 & 78 & 68 & 64 & 74 & 78 & 58 & 20 & \\
\hline 4 & 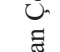 & 苛 & $\tilde{i}$ & $\stackrel{N}{I}$ & $\hat{8}$ & 76 & 84 & 74 & 72 & 54 & 74 & 84 & 54 & 30 & 22,8 \\
\hline & 灵 & & & & & 74 & 78 & 48 & 84 & 58 & 64 & 84 & 48 & 36 & \\
\hline & & & & & & 82 & 82 & 68 & 84 & 68 & 74 & 84 & 68 & 16 & \\
\hline & & & & & & 48 & 42 & 53 & 58 & 53 & 64 & 64 & 42 & 22 & \\
\hline & & & & & & 53 & 42 & 48 & 53 & 58 & 53 & 58 & 42 & 16 & \\
\hline 5 & $\stackrel{\mathbb{N}}{\stackrel{N}{\Xi}}$ & $\bar{\Xi}$ & in & $\stackrel{t}{t}$ & ô. & 42 & 48 & 64 & 58 & 58 & 58 & 64 & 42 & 22 & 21,2 \\
\hline & & & & & & 40 & 42 & 53 & 53 & 69 & 42 & 69 & 42 & 27 & \\
\hline & & & & & & 45 & 48 & 53 & 58 & 64 & 48 & 64 & 45 & 19 & \\
\hline & & & & & & 79 & 69 & 58 & 74 & 53 & 53 & 79 & 53 & 26 & \\
\hline & $\stackrel{\bigcirc}{\leftrightarrows}$ & & & & & 85 & 64 & 64 & 64 & 58 & 53 & 58 & 53 & 32 & \\
\hline 6 & Uే & $\frac{0}{\Sigma^{\pi}}$ & $\nabla$ & $\stackrel{t}{t}$ & $\begin{array}{l}n \\
0 \\
0\end{array}$ & 69 & 64 & 48 & 58 & 58 & 58 & 69 & 58 & 11 & 23,2 \\
\hline & 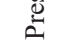 & & & & & 79 & 74 & 64 & 64 & 53 & 53 & 79 & 53 & 26 & \\
\hline & & & & & & 74 & 64 & 58 & 79 & 58 & 58 & 79 & 58 & 21 & \\
\hline
\end{tabular}


Table 3. Demographic data regarding oligosymptomatic group; breed, gender (sex), age distribution, IFAT titers, cTnI concentration and Pd analysis

\begin{tabular}{|c|c|c|c|c|c|c|c|c|c|c|c|c|c|c|c|}
\hline \multicolumn{16}{|c|}{ OLIGOSYMPTOMATIC GROUP } \\
\hline \multirow[b]{2}{*}{$\dot{z}$} & \multirow[b]{2}{*}{ 窇 } & \multirow[b]{2}{*}{ 离 } & \multirow[b]{2}{*}{ 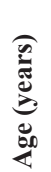 } & \multirow[b]{2}{*}{ 焉 } & \multirow[b]{2}{*}{ 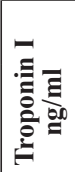 } & \multicolumn{10}{|c|}{ P- wave dispersion } \\
\hline & & & & & & I & II & III & aVR & aVL & aVF & $P$ max & $\begin{array}{c}P \\
\min \end{array}$ & Pdispersion & $\begin{array}{c}\text { mean } \\
\text { Pd }\end{array}$ \\
\hline \multirow{5}{*}{1} & \multirow{5}{*}{ 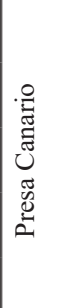 } & \multirow{5}{*}{ 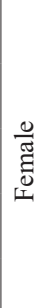 } & \multirow{5}{*}{ r } & \multirow{5}{*}{$\frac{2}{n}$} & \multirow{5}{*}{$\begin{array}{l}\tilde{\theta} \\
\hat{\theta} \\
\hat{v}\end{array}$} & 68 & 52 & 58 & 70 & 66 & 58 & 70 & 52 & 18 & \multirow{5}{*}{24} \\
\hline & & & & & & 84 & 58 & 66 & 70 & 76 & 52 & 84 & 52 & 32 & \\
\hline & & & & & & 70 & 56 & 46 & 50 & 52 & 44 & 70 & 44 & 26 & \\
\hline & & & & & & 58 & 48 & 44 & 42 & 54 & 50 & 58 & 42 & 16 & \\
\hline & & & & & & 76 & 60 & 50 & 48 & 62 & 54 & 76 & 48 & 28 & \\
\hline \multirow{5}{*}{2} & \multirow{5}{*}{ 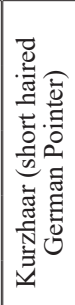 } & \multirow{5}{*}{ 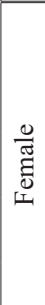 } & \multirow{5}{*}{$n^{n}$} & \multirow{5}{*}{$\stackrel{\circ}{\stackrel{\circ}{\lrcorner}}$} & \multirow{5}{*}{$\begin{array}{l}\tilde{O} \\
\hat{\nabla}\end{array}$} & 60 & 52 & 66 & 56 & 50 & 42 & 66 & 42 & 24 & \multirow{5}{*}{22} \\
\hline & & & & & & 56 & 60 & 86 & 56 & 66 & 62 & 86 & 56 & 30 & \\
\hline & & & & & & 46 & 50 & 56 & 60 & 58 & 54 & 60 & 46 & 14 & \\
\hline & & & & & & 50 & 48 & 54 & 50 & 72 & 48 & 72 & 48 & 24 & \\
\hline & & & & & & 46 & 40 & 58 & 48 & 58 & 50 & 58 & 40 & 18 & \\
\hline \multirow{5}{*}{3} & \multirow{5}{*}{ 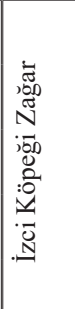 } & \multirow{5}{*}{$\sum^{\frac{0}{\pi}}$} & & & & 56 & 48 & 40 & 50 & 64 & 62 & 64 & 40 & 24 & \\
\hline & & & & & & 42 & 68 & 50 & 52 & 46 & 56 & 68 & 42 & 26 & \\
\hline & & & + & $\frac{7}{n}$ & $\stackrel{0}{0}$ & 40 & 48 & 42 & 48 & 50 & 38 & 50 & 38 & 12 & 20,8 \\
\hline & & & & & & 46 & 52 & 46 & 36 & 50 & 48 & 50 & 36 & 14 & \\
\hline & & & & & & 56 & 66 & 44 & 40 & 38 & 64 & 66 & 38 & 28 & \\
\hline & & & & & & 76 & 50 & 40 & 58 & 46 & 56 & 76 & 40 & 36 & \\
\hline & 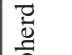 & & & & & 50 & 42 & 46 & 50 & 42 & 44 & 50 & 42 & 8 & \\
\hline 4 & ॠ & $\sum^{\frac{0}{\pi}}$ & $\sim$ & ڤn & $\begin{array}{l}0 \\
0 \\
0\end{array}$ & 48 & 56 & 50 & 46 & 58 & 52 & 58 & 46 & 12 & 20,8 \\
\hline & ह & & & & & 66 & 50 & 40 & 64 & 38 & 54 & 66 & 38 & 28 & \\
\hline & & & & & & 68 & 56 & 54 & 56 & 48 & 62 & 68 & 48 & 20 & \\
\hline & & & & & & 58 & 50 & 70 & 50 & 62 & 40 & 70 & 40 & 30 & \\
\hline &.$\varrho$ & & & & & 48 & 46 & 48 & 64 & 46 & 34 & 64 & 34 & 30 & \\
\hline 5 & $\tilde{\sigma}^{\tilde{z}}$ & $\frac{0}{\pi}$ & t & 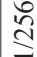 & $\stackrel{0}{0}$ & 54 & 66 & 68 & 54 & 52 & 48 & 68 & 48 & 20 & 26,8 \\
\hline & 吾 & & & & & 60 & 58 & 76 & 46 & 58 & 46 & 76 & 46 & 30 & \\
\hline & & & & & & 50 & 48 & 72 & 48 & 62 & 58 & 72 & 48 & 24 & \\
\hline & & & & & & 66 & 62 & 64 & 76 & 60 & 72 & 76 & 60 & 16 & \\
\hline & 可 $\widehat{\bar{D}}$ & & & & & 86 & 82 & 68 & 74 & 72 & 60 & 86 & 60 & 26 & \\
\hline 6 & $\frac{0}{0} \stackrel{0}{=}$ & 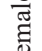 & $m$ & ڤొ & $\vec{\sim}$ & 66 & 62 & 76 & 66 & 58 & 64 & 76 & 58 & 18 & 22 \\
\hline & 造离 & & & & & 56 & 60 & 58 & 68 & 54 & 76 & 76 & 54 & 22 & \\
\hline & $\overrightarrow{\vec{a}}$ & & & & & 58 & 74 & 84 & 72 & 56 & 72 & 84 & 56 & 28 & \\
\hline
\end{tabular}


Table 4. Demographic data regarding polisymptomatic group; breed, gender (sex), age distribution, IFAT titers, cTnI concentration and Pd analysis

\begin{tabular}{|c|c|c|c|c|c|c|c|c|c|c|c|c|c|c|c|}
\hline \multicolumn{16}{|c|}{ POLYSYMPTOMATIC GROUP } \\
\hline \multirow[b]{2}{*}{$\dot{z}$} & \multirow[b]{2}{*}{ 离 } & \multirow[b]{2}{*}{ } & \multirow{2}{*}{ 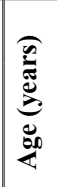 } & \multirow[b]{2}{*}{ 运 } & \multirow{2}{*}{ 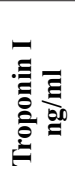 } & \multicolumn{10}{|c|}{ P-Wave dispersion } \\
\hline & & & & & & I & II & III & aVR & aVL & aVF & $\begin{array}{c}\mathbf{P} \\
\mathbf{m a x}\end{array}$ & $\begin{array}{c}\mathbf{P} \\
\min \end{array}$ & $\begin{array}{c}P \\
\text { dispersion }\end{array}$ & mean Pd \\
\hline \multirow{5}{*}{1} & \multirow{5}{*}{ 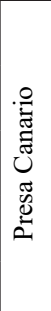 } & \multirow{5}{*}{ 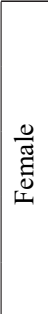 } & \multirow{5}{*}{+} & \multirow{5}{*}{$\stackrel{t}{0}$} & \multirow{5}{*}{$\frac{\pi}{0}$} & 64 & 53 & 53 & 48 & 42 & 42 & 64 & 42 & 22 & \multirow{5}{*}{24,8} \\
\hline & & & & & & 69 & 48 & 42 & 53 & 48 & 48 & 69 & 42 & 27 & \\
\hline & & & & & & 64 & 58 & 48 & 53 & 42 & 48 & 64 & 42 & 22 & \\
\hline & & & & & & 58 & 42 & 48 & 74 & 53 & 48 & 74 & 42 & 32 & \\
\hline & & & & & & 48 & 48 & 53 & 64 & 69 & 53 & 69 & 48 & 21 & \\
\hline \multirow{5}{*}{2} & \multirow{5}{*}{ 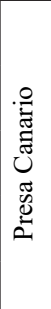 } & \multirow{5}{*}{ 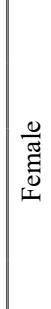 } & \multirow{5}{*}{ 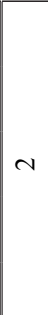 } & \multirow{5}{*}{ ڤొ } & \multirow{5}{*}{$\frac{m}{=}$} & 78 & 68 & 64 & 58 & 76 & 66 & 78 & 58 & 20 & \multirow{5}{*}{26,8} \\
\hline & & & & & & 82 & 56 & 50 & 62 & 86 & 64 & 86 & 50 & 36 & \\
\hline & & & & & & 58 & 54 & 68 & 70 & 62 & 68 & 70 & 54 & 16 & \\
\hline & & & & & & 54 & 72 & 90 & 62 & 70 & 54 & 90 & 54 & 36 & \\
\hline & & & & & & 64 & 58 & 58 & 58 & 84 & 58 & 84 & 58 & 26 & \\
\hline \multirow{5}{*}{3} & \multirow{5}{*}{ 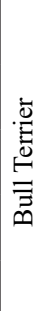 } & \multirow{5}{*}{ 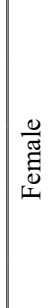 } & & & & 58 & 74 & 53 & 64 & 48 & 58 & 74 & 48 & 26 & \\
\hline & & & & & & 48 & 48 & 80 & 53 & 53 & 48 & 80 & 48 & 32 & \\
\hline & & & $\approx$ & $\stackrel{2}{n}$ & $\hat{0}$ & 74 & 74 & 74 & 69 & 69 & 63 & 74 & 63 & 11 & 23,6 \\
\hline & & & & & & 48 & 80 & 74 & 48 & 48 & 53 & 80 & 48 & 32 & \\
\hline & & & & & & 58 & 42 & 69 & 69 & 69 & 58 & 69 & 42 & 27 & \\
\hline & & & & & & 48 & 52 & 42 & 70 & 54 & 54 & 70 & 42 & 28 & \\
\hline & 过 & & & & & 46 & 42 & 64 & 62 & 56 & 46 & 64 & 42 & 22 & \\
\hline 4 & ॠ & 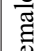 & 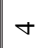 & $\frac{2}{n}$ & : & 50 & 54 & 44 & 46 & 76 & 50 & 76 & 44 & 32 & 25,6 \\
\hline & 芩 & & & & & 48 & 46 & 66 & 44 & 66 & 48 & 66 & 44 & 22 & \\
\hline & & & & & & 58 & 66 & 56 & 42 & 60 & 50 & 66 & 42 & 24 & \\
\hline & & & & & & 64 & 53 & 69 & 64 & 85 & 64 & 85 & 53 & 32 & \\
\hline & & & & & & 53 & 69 & 74 & 69 & 74 & 69 & 74 & 53 & 21 & \\
\hline 5 & $\frac{N}{\mathbb{S}}$ & $\frac{0}{\pi}$ & $r$ & $\stackrel{2}{i n}$ & $\ddot{O}_{0}$ & 48 & 69 & 69 & 69 & 64 & 58 & 69 & 48 & 21 & 27,6 \\
\hline & & & & & & 53 & 58 & 64 & 69 & 69 & 69 & 69 & 53 & 16 & \\
\hline & & & & & & 58 & 69 & 74 & 64 & 106 & 69 & 106 & 58 & 48 & \\
\hline & & & & & & 48 & 69 & 58 & 74 & 53 & 53 & 79 & 53 & 26 & \\
\hline & & & & & & 53 & 64 & 64 & 64 & 58 & 53 & 58 & 53 & 32 & \\
\hline 6 & $\frac{N}{\stackrel{N}{N}}$ & $\begin{array}{l}\text { ज्ञ } \\
\text { हुँ }\end{array}$ & in & $\underset{=}{t}$ & $\hat{0}$ & 42 & 64 & 48 & 58 & 58 & 58 & 69 & 58 & 11 & 23,2 \\
\hline & & & & & & 40 & 74 & 64 & 64 & 53 & 53 & 79 & 53 & 26 & \\
\hline & & & & & & 45 & 64 & 58 & 79 & 58 & 58 & 79 & 58 & 21 & \\
\hline
\end{tabular}


Table 5. Mean (standard deviation) cTnI and Pd values in dogs with CVL.

\begin{tabular}{|c|c|c|c|c|c|c|}
\hline \multirow{2}{*}{ Grup } & \multicolumn{2}{|c|}{$\mathrm{cTnI}(\mathrm{ng} / \mathrm{mL})$} & Referance $<0,03$ & \multicolumn{3}{|c|}{ Referance $<36$} \\
\hline & Mean & S.d & Min-Max & Ortalama & S.d & Min-Max \\
\hline Healthy Control & 0 & 0 & 0 & $22,7667^{a}$ & 3,12517 & $20,00-28,80$ \\
\hline Asymptomatic & 0,0383 & 0,04491 & $0,00-0,10$ & $22,0333^{\mathrm{ac}}$ & 0,80416 & $21,20-23,20$ \\
\hline Oligosymptomatic & 0,1685 & 0,04491 & $0,00-0,82$ & $22,7333^{\text {acd }}$ & 0,80416 & $20,80-26,80$ \\
\hline Polisymptomatic & 0,0567 & 0,05574 & $0,00-0,14$ & $25,6777^{\mathrm{b}}$ & 1,41798 & $23,60-27,60$ \\
\hline $\mathrm{P}$ values & & 0,145 & & & 0,042 & \\
\hline
\end{tabular}

* a, b, c, d; The differences between the averages with different letters in the same column are significantly $(\mathrm{p}<0.05)$. $\mathrm{p}$ value; Control and statistical comparisons of the patient groups.

\section{IFAT results}

Regarding IFAT titers, intra group comparison revealed that there was a significant difference between control and asymptomatic $(\mathrm{p}<0,05)$ groups. Among all dogs involved and subjected to IFAT analysis 12 out of $18(66,66 \%)$ presented $1 / 128$ or higher seropsitivity, $6(5,88 \%)$ at $1 / 64$ positivity (border or suspected), whereas control group were entirely negative (Table $1-5)$.

\section{Pd results}

$\mathrm{Pd}$ values (mean \pm standard deviation) in control, asymptomatic, oligosymptomatic and polisymptomatic groups were deemed as $22,76 \pm 3,12,22,03 \pm 0,80,22,73 \pm 0,80$ and $25,67 \pm 1,41 \mathrm{~ms}$, respectively. Intragroup comparison revealed significant differences among polisymptomatic group within control $(p=0,026)$, asymptomatic $(p=0,012)$ and oligosymptomatic $(\mathrm{p}=0,027)$ groups (Figure 7, Table 1-5).

\section{DISCUSSION}

$\mathrm{P}$-wave dispersion (Pd) is a biomarker as an
ECG index used both in human cardiology and veterinary medicine (20-22). It has been recognized as the difference between the maximum and the minimum $\mathrm{P}$-wave duration recorded from several ECG leads $(19,23)$. Alterations in the Pd might be presenting the prolonged intra-and inter-atrial conduction (23-25). Pd is a prognostic biomarker for detecting atrial fibrillation $(\mathrm{AF})(22,26)$. It was denoted that by determining $\mathrm{Pd}$, there would be a probability to evaluate cases not presenting visible cardiac disorders although having an increased risk for development of AF. Regarding veterinary medicine, up to 2008 the $\mathrm{Pd}$ has been evaluated only on healthy dogs (20), whereas afterwards studies subjected the relationship among Pd and cardiac disorders $(20,27,28)$.

Taking into account the supprotive role of ECG for diagnosing mycoarditis in CVL (6), a detailed literature search revealed lacking data regarding ECG examination in CVL. A recent survey indicated left atrial and ventricular enlargement and to those of myocaridal hypoxia in some of the animals 
infected with CVL (25). Another study was focused on evaluation of the cardiotoxic effects of $75 \mathrm{mg} / \mathrm{kg}$ meglumine antimoniate sc for 60 days in 28 dogs with leishmaniasis. Electrocardiographic monitoring (before the onset and at the end of treatment) and serum cTnI concentrations were determined. No abnormality was detected in routine and $24 \mathrm{~h}$ electrocardiographic tracings before and after treatment. There was no statistical difference determined between serum cTnI concentrations, nor evidence of electrocardiographic features of cardiac toxicity in dogs with CVL treated with meglumine antimoniate (26). None of the studied pointed out Pd analysis. To this context a prior and important study established Pd values among 21 healthy dogs of various breeds and of both sexes (body weights ranging from 3- $70 \mathrm{~kg}$ ) at the age of 1-12 years. All cases submitted to the ECG examinationin a standing position with BTL SD-8 electrocardiographic device. In that study P-wave duration was standardly calculated in six ECG leads (I, II, III, aVR, aVL, aVF) from five cardiac cycles. The mean Pmax, Pmin and Pd were detected as55.8 $\pm 11.3 \mathrm{~ms}$, $38.9 \pm 12.5$ and $16.9 \pm 9.4$, respectively. Consequently researchers concluded proper Pd upto $36 \mathrm{~ms} \mathrm{(22).}$

Same researcher group was involved in another study with the purpose of detecting proper value of $\mathrm{Pd}$ in healthy dogs (group I, $\mathrm{n}=53$ ), chronic valvular diseased dogs (group II, $n=23$ ) and dogs with supraventricular conduction disturbances of (group III, $n=12$ ) from various breeds, of both sexes and from different body weight $(1,5-80 \mathrm{~kg})$, at the age of 0,5-17 years. All cases were subjected to ECG examination in a standing position with BTL SD-8 electrocardiographic device. P-wave duration was calculated in 9 ECG leads (I, II, III, aVR, aVL, aVF, V1, V2, V4) from 5 cardiac cycles. Among healthy dogs the proper P-wave dispersion was found as up to $24 \mathrm{~ms}$. Pd was significantly $(\mathrm{p}<0.01)$ elevated in dogs with chronic valvular diseased and dogs with supraventricular conduction disturbances (23).

In a prior study in Turkey CVL infected dogs were subjected to 12-lead ECG examination. In that limited research authors involved solely oligosymptomatic dogs and found $\mathrm{Pd}$ as $34 \pm 7.76 \mathrm{~ms}$ (27). In the present study mean \pm standard deviation of Pd values were $22.76 \pm 3.12, \quad 22.03 \pm 0.80,22.73 \pm 0.80$ and $25.67 \pm 1.41$ in the control, asymptomatic, oligosymptomatic and polysymptomatic groups, respectively. Intragroup comparison revealed that polysymptomatic group was significantly different than control $(p=0.026)$, asymptomatic $(p=0.012)$ and oligosymptomatic $(p=0.027)$ groups. Those findings might be briefly suggested that even if increased $\mathrm{Pd}$ values were comperatively evaluated among different stages of the infection with evidence of progression of the disease caused increased vcalues. It may also be claimed that $\mathrm{Pd}$ values interpreted along with cTnI might be a predictor of myocardial injury or arrythmia such as atrial fibrillation. Pd might have predictive valuein dogs with chronic valvular diseases, supraventricular conduction disturbances, dilated cardiomyopathy, or enlarged atria due to mitral/tricuspid insufficiency (17). There- 
fore it may not be unwise to draw conclusion that earlier detection dogs under arrythmia risk such as those in dogs with CVL, causing myocarditis $(6,9)$ and other cardiac problems $(5,8)$, may have helped prevention of the disease by early medical interventions.

In the present study as detected from 6 different derivations (I, II, III, aVR, aVL, aVF), especially within polisymptomatic dogs, mean Pd values were increased in comparison to healthy controls, whereas might be seen in reference ranges such as Pd 16.9 $\pm 9.4 \mathrm{~ms}$ (20) or upper limit as $24 \mathrm{~ms}$ (23) previously detected in healthy dogs. On the other hand it should be taken into account that solely 6 dogs met inclusion criteria, warranting larger population studies.

cTn have long been recognized biomarkers with high sensitivity and specificity for myocardial degeneration in man and animals. In literature few studies are available detecting troponin concentrations among CVL infected dogs.In prior retrospective study, serum cTnI concentration was measured in dogs with leishmaniasis. Investigators detected reference ranges as $>0.06 \mu \mathrm{g} / \mathrm{L}$ increased for c TnI concentration and compared among dogs with and without anemia, azotemia, and proteinuria. In 40 dogs with CVL median cTnI concentration was higher $(P=.011)$ than in 11 control dogs. Sixteen cases $(40 \%)$ with CVLpresented elevated cTnI concentration.In contrast to nonproteinuric dogs, cTnI levels were higher $(P=.017)$ in proteinuric dogs $(28)$. The latter findings indicated that increased serum cTnI concentration was indicative of cardiac injury in CVL infection (28). In another study dogs with a precise diagnosis of CVL were subjected to meglumine an timoniat+allopurinoleorallopurinolein which serum cTnI concentration were not above reference ranges $(>0.5 \mathrm{ng} / \mathrm{mL})$ prior to treatment, and posttreatment days 14 . and 28 (30).In the present study increased cTnI concentrations were detected among 10 out of $18 \mathrm{CVL}$ infected dogs to those of asymptomatic (3/6), oligosymptomatic $(3 / 6)$, polisymptomatic (6/6) groups. The mean values of $0.03 \pm 0.04, \quad 0.16 \pm 0.04$ and $0.05 \pm 0.05 \mathrm{ng} / \mathrm{dL}$ were determined in asymptomatic, oligosymptomatic, polisymptomatic groups, respectively. Inter group comparison did not reveal sstatistical significance $(p>0,05)$ wehereas individual elevations might be briefly explained within some degree of myocaridal injury.

\section{CONCLUSIONS}

As myocarditis and arrhythmias may occur in both humans and dogs with Leishmaniasis, in an attempt to evaluate ECG changes, especially assessing combination of Pd and cTnI concentration, is essential. In this study, the evaluation of this two parameters together, especially the assessment made according to stages in infected dogs CVL, is important. In human medicine, serum cTnI concentrations is used as an important marker to detect myocardial ischemia and necrosis, and it may also be reported that this parameter have high sensitivity and specificity in animals with primary or secondary cardiac disorders. Although a statistically significant difference was not found between CVL positive and control dogs in the present 
study, it was suggested that the individual increase may be associated with myocarditis due to disease. Besides, it may be claimed that the mean $\mathrm{Pd}$ values determined in especially polysymptomatic dogs was higher compared to the control group, whereas this may be accepted in the reference ranges based on mean $\mathrm{Pd}$ values reported in healthy dogs. However considering infected dogs population as 6 in each group, it may be safely claimed that further investigations regarding greater number of cases the may be warranted.

\section{ACKNOWLEDGEMENT}

The present study was summarized from MS thesis and was funded by Adnan Menderes University Research Funding Unit (ADU BAP) with project no VTF-14036.

\section{REFERENCES}

1.Alvar J, Canavate C, Molina R, Moreno J, Nieto J. Canine leishmaniasis. Adv Parasitol, 2004; 57: 1-88.

2.Baneth G, Koutinas AF, Solano-Gallego L, Bourdeau P, Ferrer L. Canine leishmaniosis new concepts and insights on an expanding zoonosis: Part one. Trends Parasitol, 2008; 24: 324-330.

3.Font A, Durall N, Domingo M, Closa JM, Mascort J, Ferrer L. Cardiac tamponade in a dog with visceral leishmaniosis. J Am Anim Hosp Assoc. 1993; 29: 95-100.

4.Zabala EE, Ramírez OJ, Bermúdez V. Leishmaniasis visceral em um canino. Rev Fac Cienc Vet Univ Cent Venez. 2005;46: 43-50. 5.Torrent E, Leiva M, Segalés J, Franch J, Peña T, Cabrera B, Pastor J. Myocarditis and generalised vasculitis associated with leish- maniosis in a dog. J Small Anim Pract. 2005; 46: 549-552.

6.Lopez-Pena M, Aleman N, Munoz F, Fondevila D, Suarez ML, Goicoa A, Nieto JM. Visceral leishmaniasis with cardiac involvement in a dog a case report. Acta Vet Scand. 2009;51: 20 .

7.Alves GBB, Pinho FA, Silva SMMS, Cruz MSP, Costa FAL. Cardiac and pulmonary alterations in symptomatic and asymptomatic dogs infected naturally with Leishmania chagasi. Braz J Med Biol Res. 2010; 43: 310-315.

8.Rosa FA, Leite JHAC, Braga ET, Moreira PRR, Baltazar FH, Biondo AW, Padua PPM, Vasconcelos RO, Camacho AA, Ferreira WL, Machado GF, Marcondes M. Cardiac Lesions in 30 Dogs Naturally Infected With Leishmania infantum chagasi. Vet Pathol. 2014; 51(3): 603-606.

9.Dos Santos FP, Pascon JPE, Pereira DTP, Anjos BL, Mistieri MLA, Silveira ID, Porciuncula ML. Clinical and histopathological features of myocarditis in dogs with visceral leishmaniasis. Arq Bras Med Vet Zootec. 2015;67(6): 1519-1527.

10.Abranches P, Santos-Gomes G, Rachamim N, Campıno L, Schnur Lf, Jaffe Cl.An experimental model for canine visceral leishmaniasis. Parasit Immunol. 1991; 13(5): 537-550.

11.Assis J, Queiroz NM, Silveira RC, Nunes CM, Oliveira TM, Junior AC, Neves MF, Machado RZ, Buzetti WA. Estudocomparativo dos métodosdiagnósticos para leishmaniose visceral emcãesoriundos de IlhaSolteira, SP. Rev Bras Parasitol Vet. 2010; 19: 17-25. 
12.de Queiroz NM, Assis JD, Oliveira TM, Machado RZ, Nunes CM, Starke-Buzetti WA. Canine visceral leishmaniasis diagnosis by immunohistochemistry and PCR in skin tissues in association with RIFI and ELISAtest. Rev Bras Parasitol Vet. 2010; 19(1): 3238.

13.de Queiroz NMGP, da Silveira RCV, de Noronha ACF, Oliveira TMFS, Machado RZ, Starke-Buzetti WA. Detection of Leishmania (L.) chagasi in canine skin. VetParasitol. 2011;178(1): 1-8.

14.Ciaramella P, Oliva G, De luna R, Grandoni L, Ambrosio R, Cortese L, Scalone A, Persechino A. A retrospective clinical study of canine leishmaniasis in 150 dogs naturally infected by Leishmania infantum. Vet Rec. 1997; 141: 539-543.

15.Moreira M, Luvizotto M, Garcia J, Corbett C, Laurenti M. Comparison of parasitological, immunological and molecular methods for the diagnosis of leishmaniasis in dogs with different clinical signs. VetParasitol. 2007;145: 245-252.

16. Coelho EA, Ramírez L, Costa MA, Coelho VT, Martins VT, Chávez-Fumagalli MA, Abánades DR. Specific serodiagnosis of canine visceral leishmaniasis using Leishmania species ribosomal protein extracts. ClinVacImmunol. 2009; 16(12): 1774-1780.

17.Noszczyk-Nowak A, Szałas A, Pasławska U, Nicpoń J. Comparison of P-wave dispersion in healthy dogs, dogs with chronic valvular disease and dogs with disturbances of supraventricular conduction. Acta Vet Scand. 2011; 53(1): 18.

18.Kraus MS, MoiseNS, Rashniw M, Dykes
N, Erb HN. Morphology of ventricular arrhythmias in the boxer as measured by 12 lead electrocardiography with pace-mapping comparison. J VetIntMed. 2002; 16: 153158.

19.Dilaveris PE, Färbom P, Batchvarov V, Ghuran A, Malik M. Circadian Behavior of P-Wave Duration, P-Wave Area, and PR Interval in Healthy Subjects. Ann Noninvasive Electrocardiol. 2001; 6(2): 92-97.

20.Noszczyk-Nowak A, Pasławska U, Szałas A, Nicpoń J. P-wave dispersion in healthy dogs. A preliminary study. Bull Vet Inst Pulawy. 2008;52: 683-688.

21.Villani GQ, Piepoli M, Rosi A, Capucci A. P-wave dispersion index: a marker of patients with paroxysmal atria fibrillation. Int $\mathrm{J}$ Cardiol. 1996; 55: 169-175.

22.Dilaveris PE, Gialafos EJ, Andrikopoulos GK, Richter DJ, Papanikolaou V, Poralis K, Gialafos JE. Clinical and electrocardiographic predictors of recurrent atrial fibrillation. Pacing Clin Electrophysiol. 2000;23: 352358 .

23.Noszczyk-Nowak A, Szałas A, Pasławska U, Nicpon J. Comparison of P-wave dispersion in healthy dogs, dogs with chronic valvular disease, and dogs with disturbances of supraventricular conduction. Acta VetScand. 2010; 53:18.

24.Noszczyk-Nowak A. P-wave dispersion in prediction of maintenance of sinus rhyt$\mathrm{hm}$ after an electrical cardioversion of atrial fibrillation in dogs.Bull Vet Inst Pulawy. 2012;56: 99-102.

25.Sousa MG, Carareto R, Silva JG, Oliveira J. Assessment of the electrocardiogram in dogs with visceral leishmaniasis. Pesqui Vet 
Bras. 2013;33(5): 643-647.

26.Luciani A, Sconza S, Civitella C, Guglielmini C. Evaluation of the cardiac toxicity of N-methyl-glucamine antimoniate in dogs with naturally occurring leishmaniasis. Vet J. 2013;196: 119-121.

27.Ural K, Nakipoğlu D, Balıkçı C. Canine visceral leishmaniasis'te $\mathrm{P}$ dalga dispersiyonu. X. Ulusal Veteriner Iç Hastalıkları Kongresi Kapadokya, Nevşehir Türkiye 27 - 30 Haziran. 2013, poster bildirisi.

28. Silvestrini P, Piviani M, Alberola J, Rodrigues-Cortes $\mathrm{A}$, Planellas $\mathrm{M}$, Roura $\mathrm{X}$, O'Brein PJ, Pastor J. Serum cardiac troponin I concentrations in dogs with leishmaniasis: correlation with age and clinicopathologic abnormalities. Vet ClinPathol. 2012; 41(4): 568-574.

29. Mendes RS, GurjãoI TA, Oliveira LM, Santana VL, Tafuri WL, Santos JRS, Dantas AFM, Souza AP. Chronic myocarditis in a dog naturally infected by Leishmania infantum chagasi: clinical and pathological aspects. ArqBras Med Vet Zootec. 2014; 66(1): 7984.

30.Xenoulis PG, Saridomichelakis MN, Chatzis MK, Kasabalis D, Petanides T, Suchodolski JS, Steiner JM. Prospective evaluation of serum pancreatic lipase immunoreactivity and troponin I concentrations in Leishmania infantum-infected dogs treated with meglumine antimonate. Vet Parasitol. 2014; 203(3-4): 326-330. 